\title{
Effect of Inflation on Two Storage Inventory Model with Time Dependent Deteriorating Items and Stock Dependent Demand
}

\author{
Preeti Sharma \\ Department of Mathematics, \\ Jiwaji University, Gwalior, India. \\ E-mail: preetibhatele@gmail.com \\ Sanjay Sharma \\ Department of Mathematics, \\ Ajay Kumar Garg Engineering College, Ghaziabad, India. \\ Corresponding author: sanjaysharma698@gmail.com \\ B. B. Singh \\ Department of Computer Science, \\ Government K. R. G. Autonomous (P. G.) College, Gwalior, India. \\ E-mail: bbsinghkrg@gmail.com \\ Anand Tyagi \\ Department of Mathematics, \\ Ajay Kumar Garg Engineering College, Ghaziabad, India. \\ E-mail: dranandtyagi@gmail.com
}

(Received March 2, 2019; Accepted February 10, 2020)

\begin{abstract}
For deteriorating product, there always be a pressure on the company to maximize the profit. In the present model, an effort is made to develop a production inventory control model having two separate warehouses. In most of the study, the demand is assumed as time dependent which is not appropriate; but here authors have considered stock based demand. An approach is taken into consideration that deterioration rate different for different warehouses.
\end{abstract}

Keywords- Warehouses, Inflation, Stock based demand, Variable rate of deterioration.

\section{Introduction}

Inventory is mostly based on the demand pattern, it may be deterministic or probabilistic. If the demand is more than inventory or items in stock, it leads to shortages or stock out. Shortage can be categorized into two different ways, first case is known as back order or backlogging in which demand is not fulfilled by the retailers. For example, if a customer goes to buy some books and if books are not available at that time then he can postpone purchasing for some time and he depend upon the shopkeeper to provide the books as soon as possible. Hence, shortages are allowed with partial backlogging case. The second one is called lost sales case where the demand is completely lost. For example, electronic equipment and gadgets etc. have very short life span due to innovative changes in the models and technology. In practise customer may be prepared to postpone purchase of some items such as fashionable item, electronic item etc. But for certain items such as medicines or food items customer will not wait and purchase them from alternative sources. 
International Journal of Mathematical, Engineering and Management Sciences

Vol. 5, No. 3, 544-555, 2020

https://doi.org/10.33889/IJMEMS.2020.5.3.045

\section{Introduction}

Deterioration is the process of decay or damages to the items, such item may become unusable for original purposes. In the present study, authors have considered the deterioration as time dependent while in many studies, deterioration assumed constant which is not feasible. If authors look at the past then the study to justify the deteriorating inventory items was first time originally studied by Ghare (1963) where many phases of inventory control models was discussed and derived an updated form of Economic Order Quantity (EOQ) model and in this attempt author assume demand as exponentially decay. Covert and Philip (1973) developed an EOQ based model for deteriorating items under the effect of Weibull distribution with partial backlogging again, this study is more explore by Adler and Nanda (1974) for optimal lot size determination multiple product case and presented an inventory control model under the effects of the learning.

Under a new approach of linear trend in demand, Bierman and Thomas (1977) presented an inventory replenishment policy for inventory decisions under inflationary condition, Choi and Hwang (1986) developed and study continuously distributed time-lags and optimization of production planning problem. For finite uniform production rate and considering demand as linearly increasing with finite uniform production rate, Hong et al. (1990) discussed production policies to maximize the profit.

By considering, the effect of finite replenishment rate and shortages, Goswami and Chaudhuri (1991) presented an EOQ model for decaying items with an effect of linear increasing trend in demand. By considering two levels of storage, an attempt for inventory control product under decaying items and considering linear pattern in demand, was discussed by Goswami and Chaudhuri (1992). Bhunia and Maiti (1994) developed and presented a model based on two warehouses for a linear increasing pattern in demand. Time varying is one of the most important approach in the field of inventory also Giri and Chaudhuri (1997) developed heuristic inventory control model for spoilage items under time-varying demand and the effect, due to shortages are also considered.

To present inventory control techniques for two warehouses based deteriorating items, Bhunia and Maiti (1998) developed a model by considering time based demand. Lee and Ying (2000) analysed a model to control inventory and to optimize the profit for deteriorating products with two-warehouse by taking time based demands. Time varying approach also considered by Goyal and Giri (2003) to explain the production based inventory model where time dependent demand was assumed.

Chellappan and Nataraja (2011) presented an model and gives a fuzzy rule by considering perishable pattern and presented a production-inventory system, For multi-warehouse based systems to determine a fixed and space leasing contracts, Hariga (2011) presented an inventory model, Singh and Saxena (2012) also explore many things in the field of inventory and presented a returned policy based model to justify backorders for a reverse logistics approach. Dem and Singh (2013) considering effect of quality and put an effort to establish a production model for highly deteriorating products.

Forghani et al. (2013) presented a single inventory cycle based demand model by considering price adjustment. The concept of limited storage was a new technology in the area of inventory control. By considering limited storage, Sharma and Singh (2013a) presented an EPQ model of decaying product based inventory with linear exponentially increasing demand rate. Sharma and 
International Journal of Mathematical, Engineering and Management Sciences

Vol. 5, No. 3, 544-555, 2020

https://doi.org/10.33889/IJMEMS.2020.5.3.045

Singh (2013b) developed an inventory model by taking multivariate consumption rate for decaying items by assuming partial backlogging where the deterioration was considered as time dependent, Singh et al. (2016) explained the preservation technology and presented a model for deteriorating products under the effect of stock based demand with trade credit period.

Sekar et al. (2017) investigated a model by applying a new concept of preservation technology and discussed an inventory control technique for deteriorating items under limited capacity storehouse, partial backlogging and the effect of inflation.

To present non-instantaneous deteriorating products, an inventory model with more effected assumptions under price sensitive demand developed by Rastogi et al. (2018) where partial backlogging of occurring shortages was also taken into account. By considering variable holding cost, Alfares and Ghaithan (2019) explained EOQ and EPQ production based inventory models for deteriorating items.

Present study is focused to develop an inventory model for two warehouses where deterioration assumed time dependent and the demand is assumed as stock dependent. For getting good results, different facilities, conditions and storage environment, here the holding cost of the inventory for both the warehouses are considered different.

\section{Assumptions and Notations}

During this study author assumes the following notations and assumptions:

$I_{\mathrm{r}}(\mathrm{t})$ : Represent retailer's positive level of the inventory in ranted warehouse $(\mathrm{RW})$.

$I_{0}(t)$ : Represent retailer's positive level of the inventory in owned warehouse (OW).

A : Stand for set up cost per for retailer.

$\mathrm{p}$ : Per unit cost of purchasing items for retailer.

$\mathrm{S} \quad$ : Per unit cost of selling price of the retailer.

W : Noted to represent the maximum stock limit of the owned warehouse (OW).

$\mathrm{C}_{\mathrm{h}_{1}}$ : Holding cost for the retailer in OW, taken as per unit time.

$\mathrm{C}_{\mathrm{h}_{2}}$ : Holding cost for the retailer in RW, taken as per unit time.

$\mathrm{s} \quad$ : Denote the stock out (shortage) cost for retailer, measured as $\$$ / unit.

$\eta$ : Denote the opportunity cost for the retailer $\$$ /unit.

$\theta$ : The rate of deterioration in $\mathrm{OW}$, where $0 \leq \theta<1$.

$\mathrm{r} \quad$ : Discount rate, offer considering time value of money where, $\mathrm{R}=\mathrm{r}-\mathrm{i}$ is a constant.

y : Lead Time of the supplier.

$t_{d}$ : Time when the inventory level is zero in RW.

$\mathrm{t}_{\mathrm{i}}$ : Denote time of zero inventory level in OW.

$\mathrm{T}$ : Inventory cycle.

In the present model to gain maximum profit some modifying assumptions are made:

(i) The demand rate $\mathrm{D}(\mathrm{t})$ which is taken as stock dependent is given by

$$
D(t)=\left\{\begin{array}{ccc}
a+b t & \text { if } & t>0 \\
a & \text { if } & t \leq 0
\end{array}\right.
$$


International Journal of Mathematical, Engineering and Management Sciences

Vol. 5, No. 3, 544-555, 2020

https://doi.org/10.33889/IJMEMS.2020.5.3.045

where, $\mathrm{b}(0 \leq \mathrm{b} \leq 1)$ is taking as a parameter to present stock dependent consumption rate and $\mathrm{a}>0$.

(ii) It is also assumes that the owned warehouse (OW) has a limited capacity of $\mathrm{W}$ units, whereas the rented warehouse (RW) has unlimited capacity.

(iii) During the study it is also assumes that the goods that are kept in OW are consumed first, and then start the goods kept in RW.

(iv) It is also assumes that, there has no deterioration in RW. No deterioration is taken or assumed at the supplier's end.

(v) In the present study we have taken, inventory cost in RW is always higher in compare to OW.

(vi) Lead time of the supplier is assumed as a random variable.

(vii) Effect of inflation and time dependent value of money are taken into account.

(viii) As to maximize the profit, unfulfilled demand is assumed backlogged where the fraction of shortages back-ordered is $\delta$, where $0 \leq \delta \leq 1$.

\section{Mathematical Model Formulation}

From the graphical representation of the model as mentioned in the Figure 1, authors can see that the inventory system. Here, out of $I_{m}$ total units, $W$ units are move to $O W$ and $\left(I_{m}-W\right)$ units are transfer to RW. As per the model, between the time intervals $\left[0, t_{d}\right]$, authors can see that the inventory level is decreasing only in the RW as a combined effect of demand as well as deterioration. For OW, inventory level is decreasing only for deterioration as there is no demand during the interval $\left[0, t_{d}\right]$ also between the time intervals $\left[t_{d}, t_{1}\right]$, the inventory level is start dropping (decaying) to zero due to overall effect of demand also during the same time interval deterioration also takes place. After completing one cycle, similar method is going on for the next cycle.

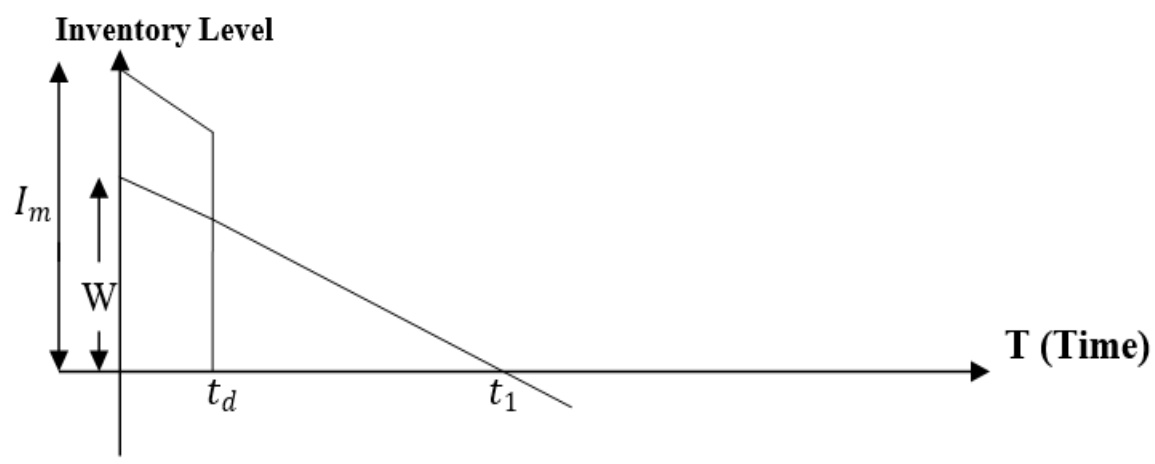

Figure 1. Graphical representation of the model

Therefore, the mathematical transformation of the model for OW during one cycle $(0 \leq \mathrm{t} \leq \mathrm{T})$ can be expressed in the term of the following differential equations (Figure 1)

$$
\begin{array}{ll}
\mathrm{I}_{0}^{\prime}(\mathrm{t})=-\theta \mathrm{tI}_{0}(\mathrm{t}), & 0 \leq \mathrm{t} \leq \mathrm{t}_{\mathrm{d}} \\
\mathrm{I}_{0}^{\prime}(\mathrm{t})+\theta \mathrm{tI}_{0}(\mathrm{t})=-(\mathrm{a}+\mathrm{bt}), & \mathrm{t}_{\mathrm{d}} \leq \mathrm{t} \leq \mathrm{t}_{1}
\end{array}
$$


International Journal of Mathematical, Engineering and Management Sciences

Vol. 5, No. 3, 544-555, 2020

https://doi.org/10.33889/IJMEMS.2020.5.3.045

$\mathrm{I}_{0}^{\prime}(\mathrm{t})=-\mathrm{a} \delta, \quad \mathrm{t}_{1} \leq \mathrm{t} \leq \mathrm{T}$

Boundary conditions are

$\mathrm{I}_{0}(0)=W, \quad \mathrm{I}_{0}\left(\mathrm{t}_{1}\right)=0$. From equation (1) $\mathrm{I}_{0}^{\prime}(\mathrm{t})=-\theta \cdot \mathrm{t} \cdot \mathrm{I}_{0} \mathrm{t}, \frac{\mathrm{I}_{0}^{\prime}(\mathrm{t})}{\mathrm{I}_{0}(\mathrm{t})}=-\theta \mathrm{t}$, on integrating $\mathrm{w}$.

r. to $\mathrm{t} \log \left\{\mathrm{I}_{0}(\mathrm{t})\right\}=-\frac{\theta \mathrm{t}^{2}}{2}+\log \mathrm{C}_{1}, \mathrm{I}_{0}(\mathrm{t})=\mathrm{C}_{1} \cdot \mathrm{e}^{-\frac{\theta \mathrm{t}^{2}}{2}}$.

Using boundary condition $\mathrm{I}_{0}(0)=\mathrm{W}$

$\mathrm{C}_{1}=\mathrm{W}, \mathrm{I}_{0}(\mathrm{t})=\mathrm{W} \cdot \mathrm{e}^{-\frac{\theta \mathrm{t}^{2}}{2}}$

From equation (2)

$\mathrm{I}_{0}^{\prime}(\mathrm{t})+\theta \cdot \mathrm{t} \cdot \mathrm{I}_{0}(\mathrm{t})=-(\mathrm{a}+\mathrm{b} \cdot \mathrm{t}), \mathrm{t}_{\mathrm{d}} \leq \mathrm{t} \leq \mathrm{t}_{1}$

I.F. $=\mathrm{e}^{\int \theta \mathrm{tdt}}=\mathrm{e}^{\frac{\theta \mathrm{t}^{2}}{2}}=\mathrm{I}_{0}(\mathrm{t}) \cdot \mathrm{e}^{\theta \frac{\mathrm{t}^{2}}{2}}=-\mathrm{a}\left[\mathrm{t}+\theta \cdot \frac{\mathrm{t}^{3}}{6}\right]-\mathrm{b} \cdot \frac{\mathrm{e}^{\theta \cdot \frac{\mathrm{t}^{2}}{2}}}{\theta}+\mathrm{C}_{2}$

Using boundary condition

$\mathrm{I}_{0}\left(\mathrm{t}_{1}\right)=0=0=-\mathrm{a}\left[\mathrm{t}_{1}+\frac{\theta}{6} \cdot \mathrm{t}_{1}^{3}\right]-\frac{\mathrm{b}}{\theta} \cdot \mathrm{e}^{\theta \cdot \frac{\mathrm{t}_{1}^{2}}{2}}+\mathrm{C}_{2}, \mathrm{C}_{2}=\mathrm{a}\left(\mathrm{t}_{1}+\frac{\theta}{6} \cdot \mathrm{t}_{1}^{3}\right)+\frac{\mathrm{b}}{\theta} \cdot \mathrm{e}^{\theta \frac{\mathrm{t}_{1}^{2}}{2}}$,

Putting in (5)

$\mathrm{I}_{0}(\mathrm{t}) \cdot \mathrm{e}^{\theta \frac{\mathrm{t}^{2}}{2}}=-\mathrm{a}\left(\mathrm{t}+\theta \cdot \frac{\mathrm{t}^{3}}{6}\right)-\frac{\mathrm{b}}{\theta} \cdot \mathrm{e}^{\theta \frac{\mathrm{t}^{2}}{2}}+\mathrm{a}\left(\mathrm{t}_{1}+\frac{\theta}{6} \cdot \mathrm{t}_{1}^{3}\right)+\frac{\mathrm{b}}{\theta} \cdot \mathrm{e}^{\theta \cdot \frac{\mathrm{t}_{1}^{2}}{2}}$ that is

$\mathrm{I}_{0}(\mathrm{t})=\mathrm{a}\left[\left(\mathrm{t}_{1}+\frac{\theta}{6} \cdot \mathrm{t}_{1}^{3}\right)-\left(\mathrm{t}+\frac{\theta}{6} \cdot \mathrm{t}^{3}\right) \cdot \mathrm{e}^{-\theta \frac{\mathrm{t}^{2}}{2}}\right]+\frac{\mathrm{b}}{\theta}\left[\mathrm{e}^{\frac{\theta}{2}\left(\mathrm{t}_{1}^{2}-\mathrm{t}^{2}\right)}-1\right]$

From equation (3)

$\mathrm{I}_{0}^{\prime}(\mathrm{t})=-\mathrm{a} . \delta$

On integrating w. r. t. $\mathrm{t}$

$\mathrm{I}_{0}(\mathrm{t})=-\mathrm{a} \delta \mathrm{t}+\mathrm{C}_{3}$, using boundary condition

$\mathrm{I}_{0}(\mathrm{t})=0 \Rightarrow \mathrm{C}_{3}=\mathrm{a} \delta \mathrm{t}_{1}$

$\mathrm{I}_{0}(\mathrm{t})=\mathrm{a} \cdot \delta \cdot\left(\mathrm{t}_{1}-\mathrm{t}\right), \quad \mathrm{t}_{1} \leq \mathrm{t} \leq \mathrm{T}$

For RW the mathematical expression of the model can be expressed in the term of the following differential equations:

$\mathrm{I}_{\mathrm{r}}^{\prime}(\mathrm{t})=-[\mathrm{a}+\mathrm{b} . \mathrm{t}], \quad 0 \leq \mathrm{t} \leq \mathrm{t}_{\mathrm{d}}$ 
$I_{r}(t)=-\left(a t+b \frac{t^{2}}{2}\right)+C_{4}$

Using boundary condition $\quad I_{r}\left(t_{d}\right)=0$

$0=-\left(a \cdot t_{d}+\frac{b}{2} t_{d}^{2}\right)+C_{4}$

$\Rightarrow \mathrm{C}_{4}=\left(\mathrm{a} \cdot \mathrm{t}_{\mathrm{d}}+\frac{\mathrm{b}}{2} \cdot \mathrm{t}_{\mathrm{d}}^{2}\right)$.

$I_{r}(t)=-\left(a t+\frac{b t^{2}}{2}\right)+\left(a \cdot t_{d}+\frac{b}{2} \cdot t_{d}^{2}\right)$

$\Rightarrow I_{r}(t)=a\left(t_{d}-t\right)+\frac{b}{2}\left(t_{d}^{2}-t^{2}\right)$

The ordering quantity based on replenishment cycle, calculated as

$$
\begin{aligned}
& \theta=\mathrm{I}_{0}(0)+\mathrm{I}_{\mathrm{r}}(0)-\mathrm{I}_{0}(\mathrm{~T}), \\
& \mathrm{I}_{\mathrm{m}}=\theta=\mathrm{W}+\left(\mathrm{a} \cdot \mathrm{t}_{\mathrm{d}}+\frac{\mathrm{b}}{2} \cdot \mathrm{t}_{\mathrm{d}}^{2}\right)+\mathrm{a} \delta \cdot\left(\mathrm{T}-\mathrm{t}_{1}\right)
\end{aligned}
$$

Now, to present the average profit of the retailer after end of one cycle, following elements should be counted:

\section{(a) Cost of Holding the Inventory of the Retailer for RW}

From the graphical representation of the model, it is obvious that to count holding cost of carrying inventory items. Authors should calculate by considering the effect of inflation as retailer is found to be in the possession of inventory between the intervals $\left[0, t_{d}\right]$.

$$
\begin{aligned}
& C_{R W}=C_{h_{2}} \cdot \int_{0}^{t_{d}} I_{r}(t) \cdot e^{-R t} \cdot d t \\
& =C_{h_{2}}\left[a \cdot \left\{-\frac{t_{d}}{R} \cdot\left(e^{-R \cdot t_{d}}-1\right)+\frac{t_{d}}{R} \cdot e^{-R \cdot t_{d}}-\frac{1}{R^{2}}\left(e^{-R \cdot t_{d}}-1\right)\right.\right. \\
& \left.\quad+\frac{b}{2}\left\{-\frac{t_{d}^{2}}{R}\left(e^{-R \cdot t_{d}}-1\right)+\frac{t_{d}^{2}}{R} \cdot e^{-R t_{d}}+\frac{2 t_{d}}{R^{2}} \cdot e^{-R \cdot t_{d}}+\frac{2}{R^{3}}\left(e^{-R t_{d}}-1\right)\right\}\right] \\
& \Rightarrow C_{R W}=C_{h_{2}} \cdot\left[a\left\{\frac{t_{d}}{R}\left(1-e^{-R \cdot t_{d}}\right)+\frac{t_{d}}{R} \cdot e^{-R \cdot t_{d}}+\frac{1}{R^{2}}\left(1-e^{-R \cdot t_{d}}\right)\right\}\right. \\
& \left.\quad+\frac{b}{2}\left\{\frac{t_{d}^{2}}{R}\left(1-e^{-R \cdot t_{d}}\right)+\frac{t_{d}^{2}}{R} e^{-R \cdot t_{d}}+\frac{2 \cdot t_{d}}{R} \cdot e^{-R \cdot t_{d}}+\frac{2}{R^{3}}\left(e^{-R \cdot t_{d}}-1\right)\right\}\right]
\end{aligned}
$$


International Journal of Mathematical, Engineering and Management Sciences

Vol. 5, No. 3, 544-555, 2020

https://doi.org/10.33889/IJMEMS.2020.5.3.045

\section{(b) Cost of Holding the Inventory of the Retailer for the Own Warehouse}

With the help of mathematical expression of the model, it can easily see that during the interval $\left[0, t_{1}\right]$, retailer is in possession of inventory so to carrying inventory, the holding cost must be calculated for $\left[0, t_{1}\right]$ with the effect of inflation.

$$
\begin{aligned}
& \mathrm{C}_{\mathrm{OW}}=\mathrm{C}_{\mathrm{h}_{1}} \cdot\left[\int_{0}^{\mathrm{t}_{\mathrm{d}}} \mathrm{I}_{0}(\mathrm{t}) \cdot \mathrm{e}^{-\mathrm{Rt}} \mathrm{dt}+\int_{\mathrm{t}_{\mathrm{d}}}^{\mathrm{t}_{1}} \mathrm{I}_{0}(\mathrm{t}) \cdot \mathrm{e}^{-\mathrm{Rt}} \mathrm{dt}\right] \\
& =\mathrm{C}_{\mathrm{h}_{1}}\left\{\left[\int_{0}^{\mathrm{t}_{\mathrm{d}}} \mathrm{W} \cdot \mathrm{e}^{-\theta \cdot \frac{\mathrm{t}^{2}}{2}} \cdot \mathrm{e}^{-\mathrm{Rt}} \mathrm{dt}\right]+\int_{\mathrm{t}_{\mathrm{d}}}^{\mathrm{t}_{1}}\left[\mathrm { a } \left\{\left(\mathrm{t}_{1}+\frac{\theta}{6} \mathrm{t}_{1}^{3}\right)\right.\right.\right. \\
& \left.\left.\left.-\left(\mathrm{t}+\frac{\theta}{6} \mathrm{t}^{3}\right) \cdot \mathrm{e}^{-\theta \frac{\mathrm{t}^{2}}{2}}\right\}+\frac{\mathrm{b}}{\theta} \cdot\left\{\mathrm{e}^{\frac{\theta}{2}\left(\mathrm{t}_{1}^{2}-\mathrm{t}^{2}\right)}-1\right\}\right] \cdot \mathrm{e}^{-\mathrm{Rt}} \mathrm{dt}\right] \\
& \Rightarrow \mathrm{C}_{\mathrm{OW}}=\mathrm{C}_{\mathrm{h}_{1}} \cdot\left[\int_{0}^{\mathrm{t}_{\mathrm{d}}}\left(1-\mathrm{Rt}-\frac{\theta \mathrm{t}^{2}}{2}\right) \mathrm{dt}+\mathrm{a} \cdot\left(\mathrm{t}_{1}+\frac{\theta}{6} \cdot \mathrm{t}_{1}^{3}\right) \cdot \int_{\mathrm{t}_{\mathrm{d}}}^{\mathrm{t}_{1}}(1-\mathrm{Rt}) \cdot \mathrm{dt}\right. \\
& -\mathrm{a} \cdot \int_{\mathrm{t}_{\mathrm{d}}}^{\mathrm{t}_{1}}\left(\mathrm{t}-\mathrm{R \textrm {t } ^ { 2 }}-\frac{1}{3} \theta \cdot \mathrm{t}^{3}-\frac{\mathrm{R} \theta}{6} \mathrm{t}^{4}-\frac{\theta^{2}}{12} \cdot \mathrm{t}^{5}\right) \mathrm{dt} \\
& \left.+\frac{\mathrm{b}}{-\theta} \cdot \int_{\mathrm{t}_{\mathrm{d}}}^{\mathrm{t}_{1}} \frac{\theta}{2} \cdot\left(\mathrm{t}_{1}^{2}-\mathrm{t}^{2}\right) \cdot \mathrm{dt}\right] \\
& =\mathrm{C}_{\mathrm{h}_{1}} \cdot\left[\left\{\mathrm{t}-\mathrm{R} \cdot \frac{\mathrm{t}^{2}}{2}-\theta \cdot \frac{\mathrm{t}^{3}}{6}\right\}_{0}^{\mathrm{t}_{\mathrm{d}}}+\mathrm{a} \cdot\left\{\mathrm{t}-\mathrm{R} \cdot \frac{\mathrm{t}^{2}}{2} \cdot\right\}_{\mathrm{t}_{\mathrm{d}}}^{\mathrm{t}_{1}} \cdot\left(\mathrm{t}_{1}+\frac{\theta}{6} \mathrm{t}_{1}^{3}\right)\right. \\
& \text {-a. } \left.\left\{\frac{\mathrm{t}^{2}}{2}-\frac{\mathrm{Rt}^{3}}{3}-\frac{\theta \mathrm{t}^{4}}{12}-\frac{\mathrm{R} \theta \cdot \mathrm{t}^{5}}{30}-\frac{\theta^{2} \cdot \mathrm{t}^{6}}{72}\right\}_{\mathrm{t}_{\mathrm{d}}}^{\mathrm{t}_{1}}+\frac{\mathrm{b}}{2}\left\{\mathrm{t}_{1}^{2} \cdot \mathrm{t}-\frac{\mathrm{t}^{3}}{3}\right\}_{\mathrm{t}_{\mathrm{d}}}^{\mathrm{t}_{1}}\right] \\
& =\mathrm{C}_{\mathrm{h}_{1}} \cdot\left[\left(\mathrm{t}_{\mathrm{d}}-\mathrm{R} \cdot \frac{\mathrm{t}_{\mathrm{d}}^{2}}{2}-\theta \cdot \frac{\mathrm{t}_{\mathrm{d}}^{3}}{6}\right)+\mathrm{a} \cdot\left(\mathrm{t}_{1}+\frac{\theta}{6} \cdot \mathrm{t}_{1}^{3}\right) \cdot\left\{\left(\mathrm{t}_{1}-\mathrm{t}_{\mathrm{d}}\right)-\frac{\mathrm{R}}{2}\left(\mathrm{t}_{1}^{2}-\mathrm{t}_{\mathrm{d}}^{2}\right)\right\}\right. \\
& -a \cdot\left\{\frac{1}{2}\left(\mathrm{t}_{1}^{2}-\mathrm{t}_{\mathrm{d}}^{2}\right)-\frac{\mathrm{R}}{3}\left(\mathrm{t}_{1}^{3}-\mathrm{t}_{\mathrm{d}}^{3}\right)-\frac{\theta}{12}\left(\mathrm{t}_{1}^{4}-\mathrm{t}_{\mathrm{d}}^{4}\right)-\frac{\mathrm{R} \theta}{30}\left(\mathrm{t}_{1}^{5}-\mathrm{t}_{\mathrm{d}}^{5}\right)\right. \\
& \left.\left.-\frac{\theta^{2}}{72}\left(\mathrm{t}_{1}^{6}-\mathrm{t}_{\mathrm{d}}^{6}\right)\right\}+\frac{\mathrm{b}}{2}\left\{\frac{2}{3} \cdot \mathrm{t}_{1}^{3}-\mathrm{t}_{1}^{2} \cdot \mathrm{td}+\frac{\mathrm{t}_{\mathrm{d}}^{3}}{3}\right\}\right]
\end{aligned}
$$

\section{(c) The Cost of Purchasing Items of the Retailer}

Order is attempt by the retailer at $t=0$, which mean that he can made the payment based his choice only. So, there will not be any effect on lead time taken by the supplier to make payment to retailer.

$$
\mathrm{PC}=\mathrm{PI}_{\mathrm{m}}=\mathrm{P}\left[\mathrm{W}+\mathrm{a}\left(\mathrm{t}_{\mathrm{d}}\right)+\frac{\mathrm{b}}{2}\left(\mathrm{t}_{\mathrm{d}}^{2}\right)+\mathrm{a} \delta\left(\mathrm{T}-\mathrm{t}_{1}\right) \cdot \mathrm{e}^{-\mathrm{RT}}\right]
$$

\section{(d) Cost of Setup Inventory of the Retailer}

It is obvious from the model that setup cost is always made by the retailer at initial time for each period, hence: $\mathrm{SPC}=\mathrm{A}$ 
International Journal of Mathematical, Engineering and Management Sciences

Vol. 5, No. 3, 544-555, 2020

https://doi.org/10.33889/IJMEMS.2020.5.3.045

\section{(e) Shortage Cost of the Retailer}

It can also be seen from the mathematical formulation of the model during the interval $\left[\mathrm{t}_{1}, \mathrm{~T}\right]$, shortage are assumed partially backlogged by the retailer.

$$
\begin{aligned}
& \mathrm{c}_{2}=\mathrm{s} \cdot \int_{\mathrm{t}_{1}}^{\mathrm{T}}\left[-\mathrm{I}_{0}(\mathrm{t})\right] \cdot \mathrm{e}^{-\mathrm{Rt}} \cdot \mathrm{dt}=-\mathrm{s} \int_{\mathrm{t}_{1}}^{\mathrm{T}} \mathrm{a} \cdot \delta \cdot\left(\mathrm{t}_{1}-\mathrm{t}\right) \cdot \mathrm{e}^{-\mathrm{Rt}} \cdot \mathrm{dt} \\
& \Rightarrow \mathrm{c}_{2}=\frac{\mathrm{a} \cdot \mathrm{s} \cdot \delta}{\mathrm{R}^{2}}\left[-\mathrm{R} \cdot \mathrm{T} \cdot \mathrm{e}^{-\mathrm{Rt}}+\mathrm{R} \cdot \mathrm{t}_{1} \cdot \mathrm{e}^{-\mathrm{Rt}_{1}}-\mathrm{e}^{-\mathrm{rT}}+\mathrm{e}^{-\mathrm{Rt}_{1}}+\mathrm{Rt}_{1} \mathrm{e}^{-\mathrm{RT}}-\mathrm{t}_{1} \cdot \mathrm{R} \cdot \mathrm{e}^{-\mathrm{Rt}_{1}}\right]
\end{aligned}
$$

\section{(f) Cost of the Retailer when Sale is Lost}

In case when demand is not fulfilled by the retailer, an extra expense beard by the retailer as lost sale cost, therefore, during end phase of the cycle where the shortages incurred and partially lost.

$$
\mathrm{C}_{0}=\pi \int_{\mathrm{t}_{1}}^{\mathrm{T}} \mathrm{a} \cdot(1-\delta) \cdot \mathrm{e}^{-\mathrm{Rt}} \mathrm{dt}=\frac{\pi \mathrm{a} \cdot(1-\delta)}{\mathrm{R}} \cdot\left(\mathrm{e}^{-\mathrm{Rt}_{1}}-\mathrm{e}^{-\mathrm{Rt}}\right)
$$

\section{(g) Complete Sales Revenue of the Retailer}

It also can be seen that the retailer shut off his entire inventory at the time $t=0$. The revenue is obtained by the retailer from starting to end of the cycle process.

$$
\begin{aligned}
& \mathrm{SR}=\mathrm{S}\left[\int_{0}^{\mathrm{t}_{1}}(\mathrm{a}+\mathrm{b} \cdot \mathrm{t}) \cdot \mathrm{e}^{-\mathrm{Rt}} \mathrm{dt}+\int_{\mathrm{t}_{1}}^{\mathrm{T}} \delta \cdot(\mathrm{a}+\mathrm{b} \cdot \mathrm{t}) \cdot \mathrm{e}^{-\mathrm{Rt}} \mathrm{dt}\right] \\
& \Rightarrow \mathrm{SR}=\mathrm{S}\left[\frac{\mathrm{a}}{\mathrm{R}}\left(1-\mathrm{e}^{-\mathrm{Rt}_{1}}\right)+\mathrm{b} \cdot\left\{-\frac{\mathrm{t}_{1}}{\mathrm{R}} \cdot \mathrm{e}^{-\mathrm{Rt}_{1}}+\frac{1}{\mathrm{R}^{2}}\left(1-\mathrm{e}^{-\mathrm{Rt}_{1}}\right)\right\}\right. \\
& \left.\frac{\mathrm{a} \delta}{\mathrm{R}}\left(\mathrm{e}^{-\mathrm{Rt}}-\mathrm{e}^{-\mathrm{R \textrm {t } _ { 1 }}}\right)-\delta \cdot \mathrm{b}\left\{\frac{1}{\mathrm{R}}\left(\mathrm{T} \cdot \mathrm{e}^{-\mathrm{RT}}-\mathrm{t}_{1} \cdot \mathrm{e}^{-\mathrm{Rt}_{1}}\right)+\frac{1}{\mathrm{R}^{2}}\left(\mathrm{e}^{-\mathrm{Rt}}-\mathrm{e}^{-\mathrm{Rt_{1 }}}\right)\right\}\right]
\end{aligned}
$$

\section{(h) Present Worth Net Profit of the Retailer}

Net profit after the end of one cycle, generated by deducting the total expenditure (according to existence) of the retailer achieved to the sales revenue gain by the retailer.

$$
\begin{aligned}
& \mathrm{N} \cdot \mathrm{R}_{\mathrm{r}}=[\text { Sales revenue }-(\text { Total costs })] \\
& \Rightarrow \mathrm{NR}_{\mathrm{r}}\left(\mathrm{t}_{1}, \mathrm{~T}\right)=\mathrm{S}\left[\frac{\mathrm{a}}{\mathrm{R}}\left(1-\mathrm{e}^{-\mathrm{Rt}_{1}}\right)+\frac{\mathrm{b}}{\mathrm{R}_{2}}\left\{-\mathrm{t}_{1} \cdot \mathrm{R} \cdot \mathrm{e}^{-\mathrm{Rt}_{1}}+\left(1-\mathrm{e}^{-\mathrm{Rt}_{1}}\right)\right\}\right. \\
& -\frac{\mathrm{a} \cdot \mathrm{S}}{\mathrm{R}} \cdot\left(\mathrm{e}^{-\mathrm{RT}}-\mathrm{e}^{-\mathrm{R \textrm {t } _ { 1 }}}\right)-\frac{\mathrm{b}}{\mathrm{R}^{2}} \cdot\left\{\mathrm{R} \cdot\left(\mathrm{Te} \mathrm{e}^{-\mathrm{RT}}-\mathrm{t}_{1} \cdot \mathrm{e}^{-\mathrm{Rt}_{1}}\right)\right. \\
& \left.\left.+\left(\mathrm{e}^{-\mathrm{RT}}-\mathrm{e}^{-\mathrm{R \textrm {t } _ { 1 }}}\right)\right\}\right]-\mathrm{A}-\mathrm{C}_{\mathrm{h}_{1}} \cdot\left[\left(\mathrm{t}_{\mathrm{d}}-\mathrm{R} \cdot \frac{\mathrm{t}_{\mathrm{d}}^{2}}{2}-\theta \frac{\mathrm{t}_{\mathrm{d}}^{3}}{6}\right)\right. \\
& +\mathrm{a}\left(\mathrm{t}_{1}+\frac{\theta}{6} \cdot \mathrm{t}_{1}^{3}\right) \cdot\left\{\left(\mathrm{t}_{1}-\mathrm{t}_{\mathrm{d}}\right)-\frac{\mathrm{R}}{2}\left(\mathrm{t}_{1}^{2}-\mathrm{t}_{\mathrm{d}}^{2}\right)\right\}-\mathrm{a}\left\{\frac{1}{2}\left(\mathrm{t}_{1}^{2}-\mathrm{t}_{\mathrm{d}}^{2}\right)-\frac{\mathrm{R}}{3}\left(\mathrm{t}_{1}^{3}-\mathrm{t}_{\mathrm{d}}^{3}\right)\right.
\end{aligned}
$$




$$
\begin{aligned}
& \left.\left.-\frac{\theta}{12}\left(\mathrm{t}_{1}^{4}-\mathrm{t}_{\mathrm{d}}^{4}\right)-\frac{\mathrm{R} \cdot \theta}{30}\left(\mathrm{t}_{1}^{5}-\mathrm{t}_{\mathrm{d}}^{5}\right) \frac{\theta^{2}}{72}\left(\mathrm{t}_{1}^{6}-\mathrm{t}_{\mathrm{d}}^{6}\right)\right\}+\frac{\mathrm{b}}{2}\left\{\frac{2}{3} \cdot \mathrm{t}_{1}^{3}-\mathrm{t}_{1}^{2} \cdot \mathrm{t}_{\mathrm{d}}+\frac{\mathrm{t}_{\mathrm{d}}^{3}}{3}\right\}\right] \\
& -\mathrm{C}_{\mathrm{h}_{2}} \cdot\left[\mathrm{a}\left\{\frac{\mathrm{t}_{\mathrm{d}}}{\mathrm{R}}\left(1-\mathrm{e}^{-\mathrm{R} \cdot \mathrm{t}_{\mathrm{d}}}\right)+\frac{\mathrm{t}_{\mathrm{d}}}{\mathrm{R}} \cdot \mathrm{e}^{-\mathrm{R} \cdot \mathrm{t}_{\mathrm{d}}}+\frac{1}{\mathrm{R}^{2}}\left(1-\mathrm{e}^{-\mathrm{R} \cdot \mathrm{t}_{\mathrm{d}}}\right)\right\}\right. \\
& \left.+\frac{\mathrm{b}}{2}\left\{\frac{\mathrm{t}_{\mathrm{d}}^{2}}{2}\left(1-\mathrm{e}^{-\mathrm{R} \cdot \mathrm{t}_{\mathrm{d}}}\right)+\frac{\mathrm{t}_{\mathrm{d}}^{2}}{\mathrm{R}} \cdot \mathrm{e}^{-\mathrm{R} \cdot \mathrm{t}_{\mathrm{d}}}+\frac{2 \mathrm{t}_{\mathrm{d}}}{\mathrm{R}^{2}} \mathrm{e}^{-\mathrm{R} \cdot \mathrm{t}_{\mathrm{d}}}+\frac{2}{\mathrm{R}^{3}}\left(1^{-\mathrm{R} \cdot \mathrm{t}_{\mathrm{d}}}-1\right)\right\}\right] \\
& -\mathrm{p} \cdot\left[\mathrm{W}+\mathrm{a}\left(\mathrm{t}_{\mathrm{d}}\right)+\frac{\mathrm{b}}{2} \cdot \mathrm{t}_{\mathrm{d}}^{2}+\mathrm{a} \cdot \delta\left(\mathrm{T}-\mathrm{t}_{1}\right) \cdot \mathrm{e}^{-\mathrm{RT}}\right]
\end{aligned}
$$

\section{(i) Present worth Cost on Production of the Supplier}

It also seen that during the time gap $\left[\mathrm{t}_{1}, \mathrm{~T}\right]$, the supplier produces an amount of $\mathrm{I}_{\mathrm{m}}$ units, therefore, production cost of the supplier are

$$
\begin{aligned}
& P_{p}=C_{p} \cdot I_{m} \cdot \int_{t_{1}}^{T} e^{-R t} \cdot d t=C_{p} \cdot I_{m} \cdot\left[\frac{e^{-R t}}{-R}\right]_{t_{1}}^{R}=C_{p} \cdot I_{m} \cdot\left(\frac{e^{-R t_{1}}-e^{-R T}}{R}\right) \\
& \Rightarrow P_{p}=C_{p} \cdot\left(\frac{e^{-R t_{1}}-e^{-R T}}{R}\right) \cdot\left\{W+a \cdot t_{d}+\frac{b}{2} \cdot t_{d}^{2}+a \cdot \varsigma\left(T-t_{1}\right) \cdot e^{-R T}\right\}
\end{aligned}
$$

\section{(j) Present Worth Cost to Managing the Inventory of the Supplier}

Since the managing cost is always handle by the supplier, also a small increment in the cost of managing the inventory make a decrement in the time gap, stock out cost and lost sale cost, hence

$$
\text { M.C }{ }_{p}=\mu
$$

Let $\psi(\mu) \in[0,1]$ assume to represent supplier's degree of uncertainty found in the lead time. For the random variable $y$, probability density function

$$
\mathrm{f}(\mathrm{y})=\frac{1}{2 \cdot \psi(\mu) \cdot \mathrm{T}}, \quad-\psi(\mu) \mathrm{T} \leq \mathrm{y} \leq \psi(\mu) . \mathrm{T}
$$

\section{(k) Present Worth Revenue on Sales of the Supplier}

From the assumptions and formulation of model, it is obvious that total revenue which is received from the sale during demand, is transferred to the supplier's account at the initial stage of the cycle.

$\mathrm{SR}_{\mathrm{p}}=\mathrm{C} . \mathrm{I}_{\mathrm{m}}$

\section{(l) Present Worth Complete Net Profit of the Supplier}

Now the average or total profit gained by the supplier can be determined by finding the difference of sales revenue of the supplier and different costs of the supplier. 
International Journal of Mathematical, Engineering and Management Sciences

Vol. 5, No. 3, 544-555, 2020

https://doi.org/10.33889/IJMEMS.2020.5.3.045

$\mathrm{N} \cdot \mathrm{R}_{\mathrm{p}}=\mathrm{SR}_{\mathrm{p}}-\mathrm{pC}_{\mathrm{p}}-\mathrm{MC}_{\mathrm{p}}-\mathrm{SC}-\mathrm{LC}$

$\mathrm{TC}_{\mathrm{p}}$ is the total cost of the supplier, hence

$\mathrm{TC}_{\mathrm{p}}=\mathrm{pC}_{\mathrm{p}}+\mathrm{MC}_{\mathrm{p}}+\varsigma \mathrm{C}+\mathrm{LC}$

Hence, the supplier's expected profit $\mathrm{N}_{\mathrm{p}_{\mathrm{p}}}^{*}$ becomes

$N_{p_{p}}^{*}=\int_{-\infty}^{\infty} N p_{p} \cdot f(y) \cdot d y=\int_{-\infty}^{0} N p_{p} f(y) \cdot d y+\int_{0}^{\infty} N p_{p} \cdot f(y) \cdot d y$

The supplier's cost,

$\mathrm{TC}_{\mathrm{p}}^{*}=\int_{-\infty}^{0} \mathrm{~T} \cdot \mathrm{C}_{\mathrm{p}} \cdot \mathrm{f}(\mathrm{y}) \mathrm{dy}+\int_{0}^{\infty} \mathrm{TC}_{\mathrm{p}} \cdot \mathrm{f}(\mathrm{y}) \mathrm{dy}$

Now, applying maximization technique on the following problem

Max. $\mathrm{Np}_{\mathrm{p}}^{*}$, Max. $\mathrm{Np}_{\mathrm{r}}^{*}$ subject to $\mathrm{TC}_{\mathrm{p}}^{*} \leq \mathrm{X}$

\section{Conclusion}

This paper amalgamates the concepts for the product, which are scatter in two warehouses because of limited storage availability, where the effect of deterioration is considered. Authors have developed two warehouse inventory control model for the items under low life with available stock dependent demand and allowable shortages. As in the present study, an effort has been made to maximize the profit; authors have found that inventory costs in RW higher than in OW. As per the outcome of the study following major outcomes are found

(i) It is obvious that to maximize the profit and to reduce the various inventory costs associated with the study, authors must store items in OW before RW and sale the items from RW before OW.

(ii) It can also be shown through the model that to maximize the profit, stock should be transferred from the RW to the OW under a bulk base release rule.

(iii) It has been observed that there is an inverse proportionality found between profit and number of cycles.

(iv) The net profit of the supplier as well as net profit per unit time of supplier, both are calculated at the maximum level when $n=1$. The average profit with respect to unit time is decrease when a small increment is made in number of cycles.

\section{Conflict of Interest}

The authors declare that there is no conflict of interest to publish this paper. 
International Journal of Mathematical, Engineering and Management Sciences

Vol. 5, No. 3, 544-555, 2020

https://doi.org/10.33889/IJMEMS.2020.5.3.045

\section{Acknowledgements}

The authors would like to thank ROASET-2019 and Ajay Kumar Garg Engineering College, Ghaziabad for supporting with financial assistantship to continue this research.

\section{References}

Adler, G.L., \& Nanda, R. (1974). The effects of learning on optimal lot size determination-multiple product case. AIIE Transactions, 6(1), 21-27.

Alfares, H.K., \& Ghaithan, A.M. (2019). EOQ and EPQ production-inventory models with variable holding cost: state-of-the-art review. Arabian Journal for Science and Engineering, 44(3), 1737-1755.

Bhunia, A.K., \& Maiti, M. (1994). A two warehouse inventory model for a linear trend in demand. Opsearch-New Delhi, 31, 318-318.

Bhunia, A.K., \& Maiti, M. (1998). A two warehouse inventory model for deteriorating items with a linear trend in demand and shortages. Journal of the Operational Research Society, 49(3), 287-292.

Bierman Jr, H., \& Thomas, J. (1977). Inventory decisions under inflationary conditions. Decision Sciences, 8(1), 151-155.

Chellappan, C.E., \& Natarajan, M. (2011). Fuzzy rule based model for the perishable collectionproduction-inventory system. International Journal of Management Science and Engineering Management, 6(3), 183-190.

Choi, S.B., \& Hwang, H. (1986). Optimization of production planning problem with continuously distributed time-lags. International Journal of Systems Science, 17(10), 1499-1508.

Covert, R.P., \& Philip, G.C. (1973). An EOQ model for items with Weibull distribution deterioration. AIIE Transactions, 5(4), 323-326.

Dem, H., \& Singh, S.R. (2013). A production model for ameliorating items with quality consideration. International Journal of Operational Research, 17(2), 183-198.

Forghani, K., Mirzazadeh, A., \& Rafiee, M. (2013). A price-dependent demand model in the single period inventory system with price adjustment. Journal of Industrial Engineering, 2013. Article ID 593108. doi.org/10.1155/2013/593108.

Ghare, P.M. (1963). A model for an exponentially decaying inventory. Journal of Industrial Engineering, 14, 238-243.

Giri, B.C., \& Chaudhuri, K.S. (1997). Heuristic models for deteriorating items with shortages and timevarying demand and costs. International Journal of Systems Science, 28(2), 153-159.

Goswami, A., \& Chaudhuri, K. (1991). EOQ model for an inventory with a linear trend in demand and finite rate of replenishment considering shortages. International Journal of Systems Science, 22(1), 181187.

Goswami, A., \& Chaudhuri, K.S. (1992). An economic order quantity model for items with two levels of storage for a linear trend in demand. Journal of the Operational Research Society, 43(2), 157-167.

Goyal, S.K., \& Giri, B.C. (2003). The production-inventory problem of a product with time varying demand, production and deterioration rates. European Journal of Operational Research, 147(3), 549-557.

Hariga, M.A. (2011). Inventory models for multi-warehouse systems under fixed and flexible space leasing contracts. Computers \& Industrial Engineering, 61(3), 744-751. 
International Journal of Mathematical, Engineering and Management Sciences

Vol. 5, No. 3, 544-555, 2020

https://doi.org/10.33889/IJMEMS.2020.5.3.045

Hong, J.D., Sandrapaty, R.R., \& Hayya, J.C. (1990). On production policies for a linearly increasing demand and finite, uniform production rat. Computers \& Industrial Engineering, 18(2), 119-127.

Lee, C.C., \& Ying, C. (2000). Optimal inventory policy for deteriorating items with two-warehouse and time-dependent demands. Production Planning \& Control, 11(7), 689-696.

Rastogi, M., Singh, S.R., \& Kushwah, P. (2018). An inventory model for non-instantaneous deteriorating products having price sensitive demand and partial backlogging of occurring shortages. International Journal of Operations and Quantitative Management, 24(1), 59-73.

Sekar, T., Uthayakumar, R., \& Mythuradevi, P. (2017). Limited capacity storehouse inventory model for deteriorating items with preservation technology and partial backlogging under inflation. Inventory Model, 21(3), 377-404.

Sharma, S., \& Singh, S.R. (2013a). An EPQ model of deteriorating inventory with exponential demand rate under limited storage. Africa Development and Resources Research Institute, 1(1), 23-31.

Sharma, S., \& Singh, S.R. (2013b). An inventory model for decaying items, considering multi variate consumption rate with partial backlogging. Indian Journal of Science and Technology, 6(7), 4870-80.

Singh, S., Khurana, D., \& Tayal, S. (2016). An economic order quantity model for deteriorating products having stock dependent demand with trade credit period and preservation technology. Uncertain Supply Chain Management, 4(1), 29-42.

Singh, S.R., \& Saxena, N. (2012). An optimal returned policy for a reverse logistics inventory model with backorders. Advances in Decision Sciences, Article ID 386598. doi.org/10.1155/2012/386598. 